\title{
Edge stability in secure graph domination
}

\author{
Anton Pierre Burger $\|^{1}$ Alewyn Petrus de Villiers $\|^{2}$ Jan Harm van Vuuren ${ }^{2}$ \\ ${ }^{1}$ Department of Logistics, Stellenbosch University, Private Bag X1, Matieland, 7602, South Africa \\ ${ }^{2}$ Department of Industrial Engineering, Stellenbosch University, Private Bag X1, Matieland, 7602, South Africa
}

received $27^{\text {th }}$ June 2014, revised $6^{\text {th }}$ Jan. 2015, accepted $9^{\text {th }}$ Feb. 2015.

\begin{abstract}
A subset $X$ of the vertex set of a graph $G$ is a secure dominating set of $G$ if $X$ is a dominating set of $G$ and if, for each vertex $u$ not in $X$, there is a neighbouring vertex $v$ of $u$ in $X$ such that the swap set $(X-\{v\}) \cup\{u\}$ is again a dominating set of $G$. The secure domination number of $G$ is the cardinality of a smallest secure dominating set of $G$. A graph $G$ is $p$-stable if the largest arbitrary subset of edges whose removal from $G$ does not increase the secure domination number of the resulting graph, has cardinality $p$. In this paper we study the problem of computing $p$-stable graphs for all admissible values of $p$ and determine the exact values of $p$ for which members of various infinite classes of graphs are $p$-stable. We also consider the problem of determining analytically the largest value $\omega_{n}$ of $p$ for which a graph of order $n$ can be $p$-stable. We conjecture that $\omega_{n}=n-2$ and motivate this conjecture.
\end{abstract}

Keywords: Secure domination, graph protection, edge removal.

\section{Introduction}

Let $G=(V, E)$ be a simple graph of order $n$. A set $D \subseteq V$ is a dominating set of $G$ if each vertex $v \in V-D$ is adjacent to a vertex in $D$. A dominating set $X \subseteq V$ of $G$ is secure dominating if, for each vertex $u \in V-X$, there exists a vertex $v \in N(u) \cap X$ such that the swap set $(X-\{v\}) \cup\{u\}$ is again a dominating set of $G$, where $N(u)$ denotes the open neighbourhood of $u$. In this case $v$ is said to defend $u$. A vertex $u \in V-X$ is an external private neighbour of a vertex $v \in X$ with respect to some set $X$ if $N(u) \cap X=\{v\}$ and a vertex $w \in V$ is a universal vertex of $G$ if it is adjacent all the vertices in $V-\{w\}$. The minimum cardinality of a secure dominating set of $G$ is called the secure domination number of $G$ and is denoted by $\gamma_{s}(G)$. For the graph $G_{1}$ in Figure 1 , for example, $\gamma_{s}\left(G_{1}\right)=2$. Noting that at least one vertex in $\left\{v_{2}, v_{3}\right\}$ must be included in any secure dominating set of $G_{1}$, it follows that $G_{1}$ has three minimum secure dominating sets (up to isomorphism), as shown in the figure. For the minimum secure dominating set in Figure 1 a), $v_{3}$ is defended by $v_{2}$, while $v_{4}$ and $v_{5}$ are both defended by $v_{1}$. The vertex $v_{2}$ is a universal vertex of $G_{1}$ while the vertex $v_{3}$ is an external private neighbour of $v_{2}$ with respect to the minimum secure dominating set in Figure 1 a).

A number of general bounds have been established for the parameter $\gamma_{s}(G)$ in [9], and exact values of $\gamma_{s}(G)$ have also been established for various graph classes, such as paths, cycles, complete multipartite

\footnotetext{
*Email: apburgeresun.ac.za.

†Email: antondev@sun.ac.za and vuuren@sun.ac.za.
} 


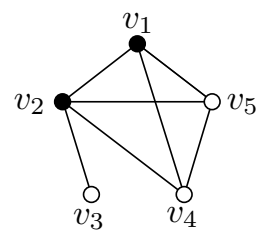

(a)

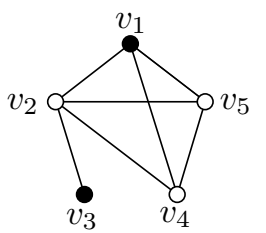

(b)

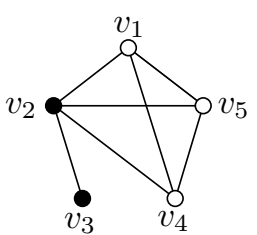

(c)

Fig. 1: All the minimum secure dominating sets (up to isomorphism) for a graph $G_{1}$ of order 5 .

graphs and products of paths and cycles. Various properties of the secure dominating sets of graphs have been studied in [1, 2, 6, 7, 8, 12].

In [4] we considered the cost (measured by the increase in the secure domination number) of deleting a number of edges from a graph. We then used our knowledge of this cost function in [3] to construct the class of all graphs $G$ of order $n$ with the property that the smallest number of arbitrary edges whose removal from $G$ necessarily increases the secure domination number of the resulting graph, is some prespecified number $q$. This problem is relevant in the generic application where the vertices of $G$ denote locations on some spatial domain, and the edges represent links between these locations along which patrolling guards may move. A secure dominating set of $G$ represents a collection of locations at which guards may be stationed so that the entire location complex modelled by $G$ is securely dominated. The studies in [3, 4] provide threshold information as to the number of edge failures (which may be caused randomly or by an adversary) that will necessitate the hiring of additional guards to secure the resulting location complex.

In this paper we focus our attention on the dual problem of determining graphs $G$ with the property that the largest number of arbitrary edges whose removal from $G$ necessarily does not increase the secure domination number of the resulting graph, is a pre-specified value $p$. This dual problem is relevant in the same generic application as described above, but where threshold information is sought with respect to the largest number of arbitrary edge failures which does not result in a requirement of additional guards to dominate the location complex securely.

The paper opens in $\S 2$ with a formalisation of the notion of $p$-stability in secure domination (with respect to edge removal). We characterise the class of $p$-stable graphs for all admissible values of $p$ in an inductive fashion in terms of $(p-1)$-stable graphs in $\S 3$. In $\S 4$ we turn our attention to the intriguing problem of determining the largest value of $p$ for which a graph of order $n$ can be $p$-stable, before determining exact values of $p$ for which paths and cycles are $p$-stable in $\S 5$. The paper closes in $\S 6$ with three suggestions for further work.

\section{The notion of $p$-stability}

We denote the set of all non-isomorphic graphs obtained by removing exactly $p \in\{0,1, \ldots, m\}$ edges from a given graph $G$ of size $m$ by $G-p e$. Similarly, $G+q e$ denotes the class of non-isomorphic graphs obtained by inserting exactly $q \in\left\{0,1, \ldots,\left(\begin{array}{l}n \\ 2\end{array}\right)-m\right\}$ edges between pairs of non-adjacent vertices of a graph $G$ of order $n$ and size $m$. Furthermore, $\gamma_{s}(G-p e)$ denotes the set of values of $\gamma_{s}(H)$ as $H \in G-p e$ varies (for a fixed value of $p$ ). The notation $\gamma_{s}(G+q e)$ has a similar meaning. We distinguish between the graph obtained by removing a specific edge $e$ from $G$, by writing $G-e$, and the class of graphs obtained 
by removing any single edge from $G$, by writing $G-1 e$. A similar distinction is made for edge insertion. It is not hard to prove the following basic result, which shows that the secure domination number of a graph cannot decrease upon removal of a subset of its edges.

Proposition $1([\mathbf{3}])$ Let $G$ be any nonempty graph and let e be any edge of $G$. Then exactly one of the following statements is true:

(a) $\gamma_{s}(G-e)=\gamma_{s}(G)$, or

(b) $\gamma_{s}(G-e)=\gamma_{s}(G)+1$.

The following result is an immediate consequence of Proposition 1

Corollary 1 For any graph $G$ of size at least $p, \gamma_{s}(G-p e) \subseteq\left\{\gamma_{s}(G), \gamma_{s}(G)+1, \ldots, \gamma_{s}(G)+p\right\}$.

It follows from Corollary 1 that the cost function

$$
C_{p}(G)=\max \gamma_{s}(G-p e)-\gamma_{s}(G)
$$

is nonnegative and bounded from above by $p$ for all $p \in\{0,1, \ldots, m\}$. This cost function measures the largest possible increase in the secure domination number of a member of $G-p e$, relative to the secure domination number of a graph $G$ of size $m$, when an arbitrary set of $p \in\{0,1, \ldots, m\}$ edges are removed from $G$. Note that a graph $G$ is $p$-stable if $C_{p}(G)=0$, but $C_{p+1}(G)>0$.

The following result shows that for any graph $G$, the associated stability value is non-increasing as more and more edges are removed from $G$, as long as the secure domination number does not change as a result of the edge removals.

Proposition 2 Let $G_{1}$ be a nonempty graph and suppose $G_{2} \in G_{1}-1$ e such that $\gamma_{s}\left(G_{1}\right)=\gamma_{s}\left(G_{2}\right)$. If $G_{1}$ is $p_{1}$-stable and $G_{2}$ is $p_{2}$-stable, then $p_{1}>p_{2}$.

Proof: Let $G_{1}$ be a $p_{1}$-stable graph and let $G_{2}$ be a $p_{2}$-stable member of $G_{1}-1 e$ such that $\gamma_{s}\left(G_{1}\right)=$ $\gamma_{s}\left(G_{2}\right)$, but suppose, to the contrary, that $p_{2}=p_{1}+p^{\prime}$ for some $p^{\prime} \in \mathbb{N}$. Then

$$
\begin{aligned}
C_{p_{2}}\left(G_{2}\right) & =\max \gamma_{s}\left(G_{2}-p_{2} e\right)-\gamma_{s}\left(G_{2}\right) \\
& =\max \gamma_{s}\left(\left(G_{2}+1 e\right)-\left(p_{2}+1\right) e\right)-\gamma_{s}\left(G_{2}\right) \\
& \geq \max \gamma_{s}\left(G_{1}-\left(p_{1}+p^{\prime}+1\right) e\right)-\gamma_{s}\left(G_{1}\right) \\
& =\max \gamma_{s}\left(\left(G_{1}-p_{1} e\right)-\left(p^{\prime}+1\right) e\right)-\gamma_{s}\left(G_{1}\right) \\
& \geq 1,
\end{aligned}
$$

contradicting the $p_{2}$-stability of $G_{2}$.

\section{Computing the class of $p$-stable graphs of order $n$}

Let $\mathcal{S}_{n}^{p}$ be the class of non-isomorphic $p$-stable graphs of order $n \geq 2$. We open this section with a characterisation of the graph class $\mathcal{S}_{n}^{p}$ in terms of the graph class $\mathcal{S}_{n}^{p-1}$ for any $p \in \mathbb{N}$. 
Proposition 3 A graph $G$ of size at least $p>0$ is p-stable if and only if

(a) $\gamma_{s}(H)=\gamma_{s}(G)$ for each $H \in G-1 e$ and

(b) each member of $G-1 e$ is $r$-stable for some $r \geq p-1$ and at least one member of $G-1 e$ is $(p-1)$-stable.

Proof: Let $G$ be a $p$-stable graph for some $p>0$. Then $\gamma_{s}(G)=\gamma_{s}(H)$ for all graphs $H \in G-1 e$, implying the necessity of (a). The necessity of (b) is established by contradiction. Suppose, contrary to (b), that there exists an $r$-stable graph $\hat{H} \in G-1 e$ for some $r<p-1$. Then,

$$
\begin{aligned}
C_{p}(G) & =\max \gamma_{s}(G-p e)-\gamma_{s}(G) \\
& =\max \gamma_{s}((G-1 e)-(p-1) e)-\gamma_{s}(G) \\
& \geq \max \gamma_{s}(\hat{H}-(p-1) e)-\gamma_{s}(\hat{H}) \\
& =C_{p-1}(\hat{H}) \\
& \geq 1
\end{aligned}
$$

contradicting the $p$-stability of $G$. This shows that all graphs in the class $G-1 e$ are $r$-stable for some $r \geq p-1$. Note, however, that by Proposition 2 there exists an $r$-stable graph $H^{\prime} \in G-1 e$ for some $r<p$. Clearly, $r=p-1$ in this case, thereby establishing the necessity of (b).

For the sufficiency of (a) and (b), suppose, to the contrary, that at least one member $H^{*} \in G-1 e$ for which $\gamma_{s}\left(H^{*}\right)=\gamma_{s}(G)$ is $(p-1)$-stable and that each member $H \in G-1 e$ for which $\gamma_{s}(H)=\gamma_{s}(G)$ is $r$-stable for some $r \geq p-1$, but that $G$ is not $p$-stable. Then $\max \gamma_{s}(G-p e)=\max \gamma_{s}(H-(p-1) e)=$ $\gamma_{s}(H)=\gamma_{s}(G)$, where the maximum is taken over all graphs $H \in G-1 e$ for which $\gamma_{s}(H)=\gamma_{s}(G)$. But $\max \gamma_{s}(G-(p+1) e) \geq \gamma_{s}\left(H^{*}-p e\right)>\gamma_{s}\left(H^{*}\right)=\gamma_{s}(G)$, contradicting the supposition that $G$ is not $p$-stable.

If the class $\mathcal{S}_{n}^{0}$ can be characterised and constructed, then the result of Proposition 3 may be used to compute the class $\mathcal{S}_{n}^{p}$ inductively from the class $\mathcal{S}_{n}^{p-1}$ for all $p \in \mathbb{N}$ and all $n \geq 2$, using the class $\mathcal{S}_{n}^{0}$ as the base case. We therefore pose the following open problem.

Problem 1 Characterise the class $\mathcal{S}_{n}^{0}$ of 0-stable graphs of order $n$.

While the above problem seems hard, it is easy to prove that $\mathcal{S}_{n}^{0}$ is nonempty for all $n \geq 2$. The next result follows immediately from a realisation that the complete graph $\mathcal{K}_{n}$ is the only graph of order $n$ with secure domination number 1 [5, Proposition 1].

Proposition $4 \mathcal{K}_{n} \in \mathcal{S}_{n}^{0}$ for all $n \geq 2$.

In fact, it follows from Proposition 4 that any graph of order $n$ which contains a nontrivial, complete component is a member of $\mathcal{S}_{n}^{0}$. The seven graphs of order 5 in Figure 2 are, for example, all members of the graph class $\mathcal{S}_{5}^{0}$ by Proposition 4.

Furthermore, each member of the graph class $\mathcal{S}_{n}^{0}$ has the following interesting property.

Proposition 5 If $G \in \mathcal{S}_{n}^{0}$ for some $n \geq 2$, then some vertex in every minimum secure dominating set $X$ of $G$ has private neighbours external to $X$. 


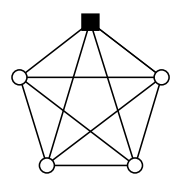

(a)

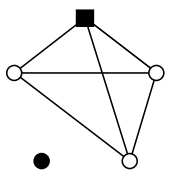

(b)

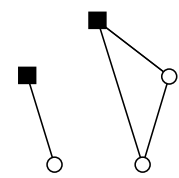

(c)

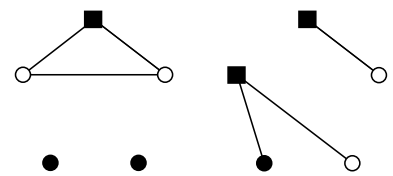

(d) (e)

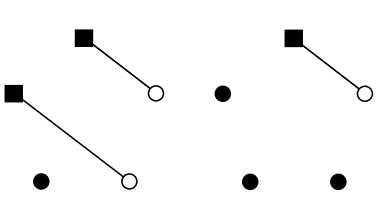

(f)

(g)

Fig. 2: A subset of the graph class $\mathcal{S}_{5}^{0}$. Minimum secure dominating sets are denoted by solid vertices in each case. Square solid vertices denote members of the minimum secure dominating set which have external private neighbours.

Proof: Suppose $G$ is 0 -stable, but suppose, to the contrary, that $G$ possesses a minimum secure dominating set $X$ in which no vertex has private neighbours external to $X$. Let $e=u v$ be any edge of $G$. Then there are three cases to consider:

Case $i: u, v \in X$. In this case, clearly, $X$ remains a secure dominating set of $G-e$, showing that $\gamma_{s}(G-e) \leq|X|=\gamma_{s}(G)$. But since $\gamma_{s}(G-e) \geq \gamma_{s}(G)$ by Proposition 1, it follows that $\gamma_{s}(G-e)=\gamma_{s}(G)$.

Case ii: $u, v \notin X$. Since $u$ and $v$ are not external private neighbours of any vertex in $X$, it follows from [9. Proposition 2] that they are not uniquely defended by a common vertex in $X$. Hence $X$ remains a secure dominating set of $G-e$, and so $\gamma_{s}(G-e)=\gamma_{s}(G)$, as above.

Case iii: $u \in X$ and $v \notin X$. Since no vertex outside $X$ is an external private neighbour of any vertex in $X, v$ has another neighbour in $X$ which does not defend any vertex outside $X$ uniquely. Hence $X$ remains a secure dominating set of $G-e$, and so $\gamma_{s}(G-e)=\gamma_{s}(G)$ yet again.

In all of the above cases, removal of the arbitrary edge $e$ from $G$ does not increase the domination number of the resulting graph, contradicting the 0-stability of $G$.

The property in Proposition 5 is, however, not sufficient to characterise the graph class $\mathcal{S}_{n}^{0}$, as illustrated by the counter example in Figure 1] The graph $G_{1}$ in the figure is not a member of $\mathcal{S}_{5}^{0}$; removal of any of its edges other than $v_{2} v_{3}$ will cause an increase in the secure domination number. Yet every minimum secure dominating set of $G_{1}$ has at least one member which possesses private neighbours external to the set, as shown in Table 1 .

\begin{tabular}{ccc}
\hline $\begin{array}{c}\text { Minimum secure } \\
\text { dominating set } X\end{array}$ & $\begin{array}{c}\text { Members of } \\
\text { the set } X\end{array}$ & $\begin{array}{c}\text { Private neighbours } \\
\text { external to } X\end{array}$ \\
\hline in Figure 1 a) & $v_{1}$ & None \\
& $v_{2}$ & $v_{3}$ \\
in Figure 1 b) & $v_{1}$ & $v_{4}, v_{5}$ \\
in Figure 1 c) & $v_{3}$ & None \\
& $v_{2}$ & $v_{1}, v_{4}, v_{5}$ \\
\hline
\end{tabular}

Tab. 1: At least one member of any secure dominating set of $G_{1}$ possesses private neighbours external to the set.

If Problem 1 can be solved and if the resulting characterisation can be used to derive an iterative or recursive construction for the graph class $\mathcal{S}_{n}^{0}$, then the inductive process of computing the graph classes $\mathcal{S}_{n}^{1}, \mathcal{S}_{n}^{2}, \mathcal{S}_{n}^{3}, \ldots$ from $\mathcal{S}_{n}^{0}$ can be achieved by Algorithm 1. The algorithm commences by systematically considering each graph $H \in \mathcal{S}_{n}^{p-1}$ and proceeding to add a single edge $e \notin E(H)$ to $H$ in Step 3, upon which the result of Proposition 3 is used to test whether $H+e$ is, in fact, $p$-stable. If $H+e$ is $p$-stable, it 
is included in the class $\mathcal{S}_{n}^{p}$. This process is repeated for each edge $e \notin E(H)$.

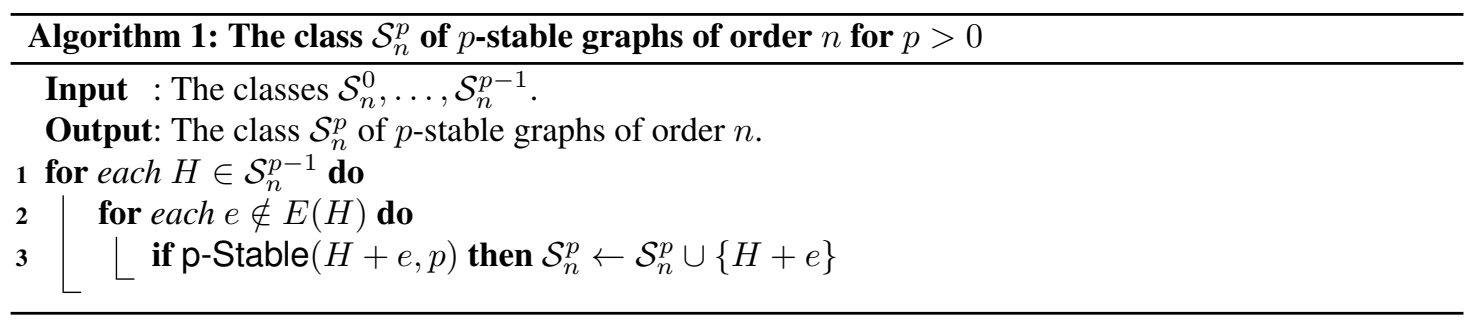

In Step 3 of Algorithm 1, another algorithm, Algorithm2 is called to test whether the graph $G=H+e$ is $p$-stable. In Algorithm 2, each member of $G-1 e$ is examined. If a member $I$ is found for which $\gamma_{s}(I) \neq \gamma_{s}(G)$, then $G$ is not $p$-stable. Furthermore, if $G-e \notin \mathcal{S}_{n}^{r}$ for $r \geq p-1$, then again $G$ is not $p$-stable by Proposition 3 .

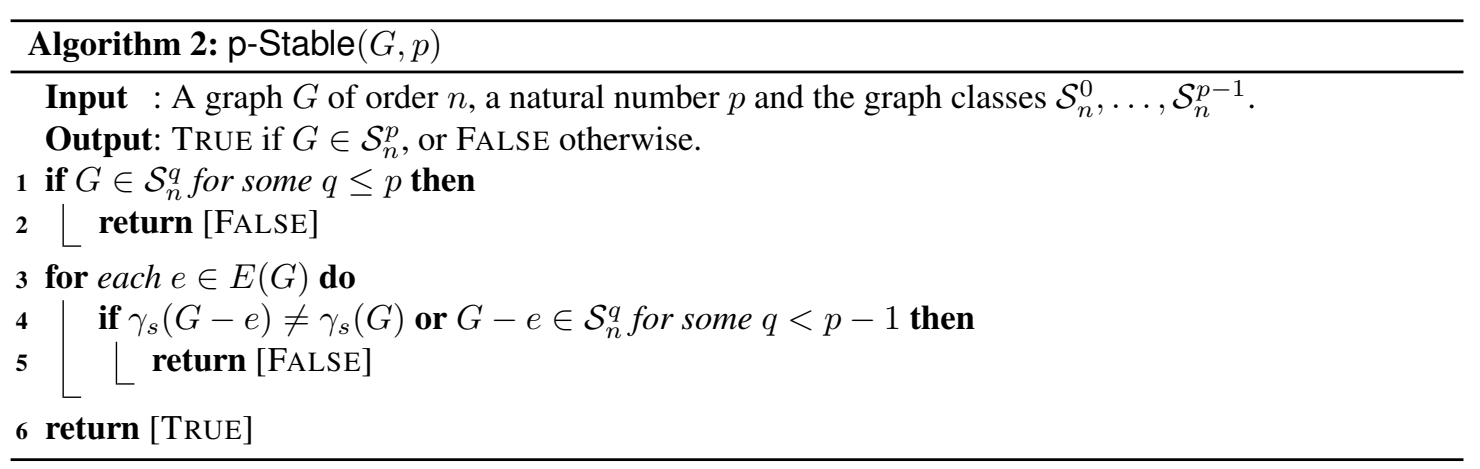

The graph classes $\mathcal{S}_{n}^{0}, \mathcal{S}_{n}^{1}, \mathcal{S}_{n}^{2}$ and $\mathcal{S}_{n}^{3}$ are shown as an example in Figure 3 . The base case, the class $\mathcal{S}_{5}^{0}$, shown in the outer layer of Figure 3 was found manually (by brute force). Thereafter, Algorithm 1 was used to compute the classes $\mathcal{S}_{5}^{1}, \mathcal{S}_{5}^{2}$ and $\mathcal{S}_{5}^{3}$ inductively. These classes are shown in the inner layers of the figure.

The cardinalities of the nonempty graph classes $\mathcal{S}_{n}^{p}$ are listed in Table 2 for all $p \in\left\{0,1, \ldots,\left(\begin{array}{l}n \\ 2\end{array}\right)\right\}$ and all $n \in\{2,3, \ldots, 9\}$.

\section{The largest $p$ for which an order $n$ graph is $p$-stable}

Let $\omega_{n}$ be the largest value of $p$ for which there exists a graph of order $n$ that is $p$-stable. It follows from Proposition 4 that $\mathcal{S}_{n}^{0} \neq \emptyset$ for all $n \geq 2$. Proposition 3 therefore implies that

$$
\mathcal{S}_{n}^{0}, \ldots, \mathcal{S}_{n}^{\omega_{n}} \neq \emptyset \text { and } \mathcal{S}_{n}^{\omega_{n}+1}, \ldots, \mathcal{S}_{n}^{\left(\begin{array}{c}
n \\
2
\end{array}\right)}=\emptyset
$$

for all $n \geq 2$. It follows from Table 2 that $\omega_{n}=n-2$ for all $n \in\{2, \ldots, 9\}$. We believe that this linear functional relation holds in general, and hence conjecture as follows. 


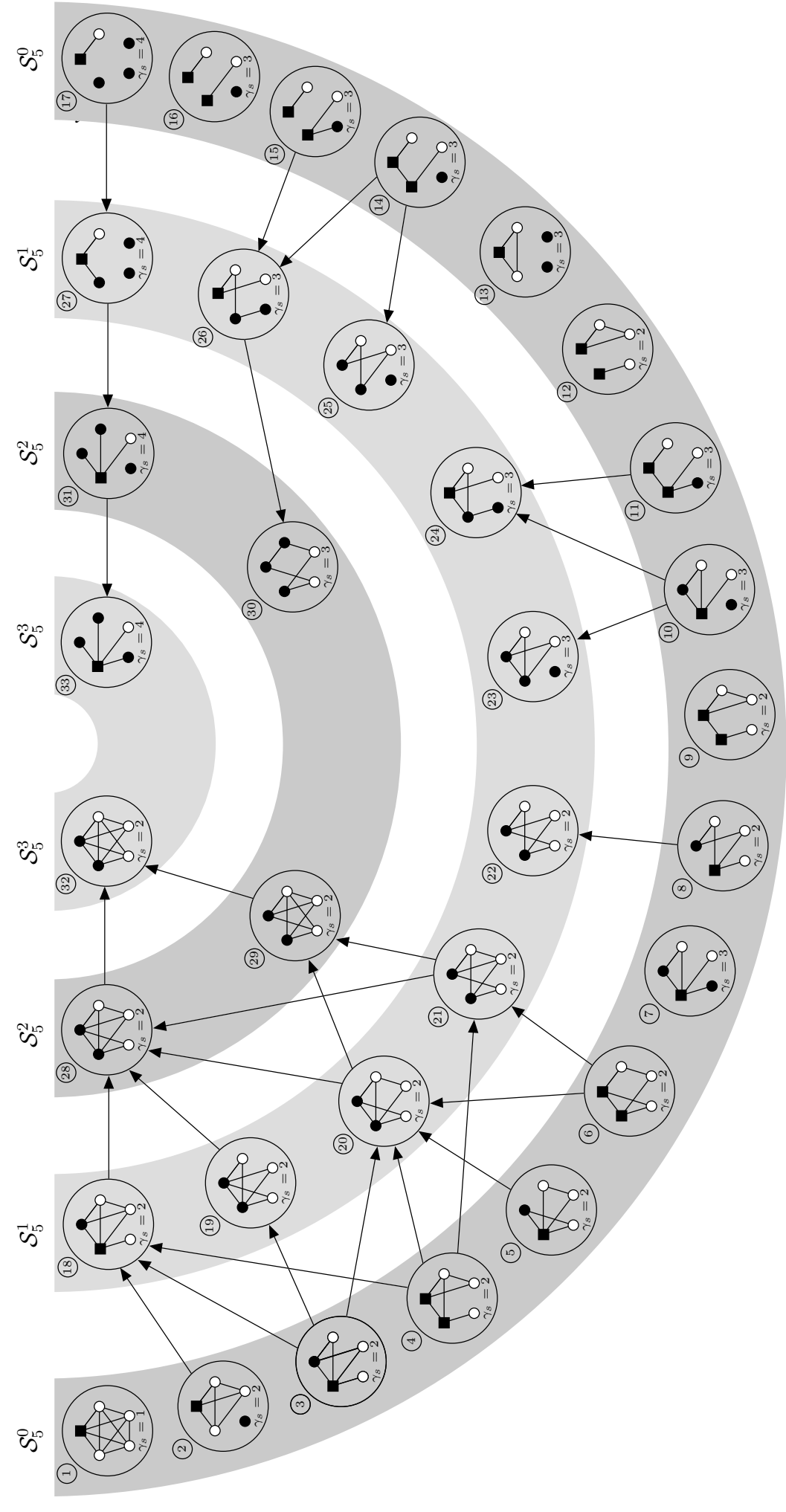

109

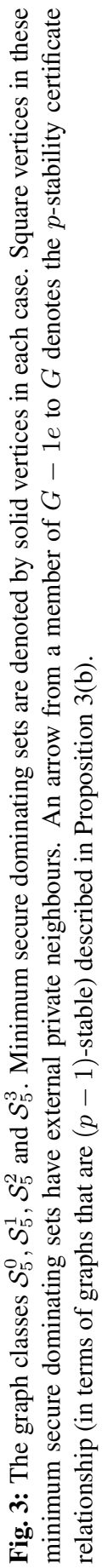




\begin{tabular}{rrrrrrrrr}
\hline$n \rightarrow$ & 2 & 3 & 4 & 5 & 6 & 7 & 8 & 9 \\
\hline$\left|\mathcal{S}_{n}^{0}\right|$ & 1 & 2 & 6 & 17 & 81 & 514 & 5460 & 107794 \\
$\left|\mathcal{S}_{n}^{1}\right|$ & & 1 & 3 & 10 & 51 & 355 & 4205 & 94106 \\
$\left|\mathcal{S}_{n}^{2}\right|$ & & & 1 & 4 & 16 & 136 & 2050 & 52502 \\
$\left|\mathcal{S}_{n}^{3}\right|$ & & & & 2 & 5 & 32 & 551 & 16923 \\
$\left|\mathcal{S}_{n}^{4}\right|$ & & & & & 2 & 5 & 70 & 3081 \\
$\left|\mathcal{S}_{n}^{5}\right|$ & & & & & & 1 & 6 & 245 \\
$\left|\mathcal{S}_{n}^{6}\right|$ & & & & & & & 3 & 13 \\
$\left|\mathcal{S}_{n}^{7}\right|$ & & & & & & & & 3 \\
\hline Total & 1 & 3 & 10 & 33 & 155 & 1043 & 12345 & 274667 \\
Time & $\ll 1$ & $<1$ & 1 & 15 & 374 & 15895 & 1069220 \\
\hline
\end{tabular}

Tab. 2: Cardinalities of the nonempty graph classes $\mathcal{S}_{n}^{1}, \ldots, \mathcal{S}_{n}^{\left(\begin{array}{c}n \\ 2\end{array}\right)}$ of orders $n \in\{2, \ldots, 9\}$ computed by a $\mathrm{C}++$ implementation of Algorithms 1 , 2 on a $3.4 \mathrm{GHz}$ Intel(R) Core(TM) i7-3770 processor with 8 GiB RAM running in Ubuntu 12.04. The Boost graph library [13] was used for isomorphism testing. The classes $\mathcal{S}_{2}^{0}, \mathcal{S}_{3}^{0}, \ldots, \mathcal{S}_{9}^{0}$ were found by brute force and the constructions of these classes were not included in the time measurements in the last row of the table (which are measured in seconds).

Conjecture $1 \omega_{n}=n-2$ for all $n \geq 2$.

Although we are unable to prove Conjecture 1, we further substantiate this conjecture and establish bounds on $\omega_{n}$ in this section.

\subsection{Upper bounds on $\omega_{n}$}

It is easy to show that $\omega_{n}$ is bounded from above by a linear function of $n$.

Proposition 6 Suppose $u$ and $v$ are two adjacent vertices of a graph $G$. Then $G$ is $p$-stable for some $p \leq \operatorname{deg}(u)+\operatorname{deg}(v)-2$.

Proof: Let $u$ and $v$ be two adjacent vertices in $G$, and let $H$ be the graph obtained by removing all the edges from $G$ that are incident to $u$ or $v$, except for the edge $u v$. Then $H$ contains $\operatorname{deg}(u)+\operatorname{deg}(v)-2$ edges fewer than $G$ and one component more than $G$. Furthermore, one of these components is isomorphic to the complete graph $\mathcal{K}_{2}$, which is 0 -stable by Proposition 4 . Hence it is possible to increase the secure domination number of $G$ by removing more than $\operatorname{deg}(u)+\operatorname{deg}(v)-2$ edges, and so $G$ is not $p$-stable for any $p \geq \operatorname{deg}(u)+\operatorname{deg}(v)-1$.

The following three consequences immediately follow from Proposition 6

Corollary 2 A connected graph with maximum degree $\Delta$ is $p$-stable for some $p \leq 2(\Delta-1)$.

Corollary 3 A connected graph with minimum degree $\delta$ and maximum degree $\Delta$ which contains a universal vertex is p-stable for some $p \leq \Delta+\delta-3$.

Corollary $4 \omega_{n} \leq 2 n-4$ for all $n \geq 2$.

When further restrictions are placed on the graph, then improvements on the above bounds, such as the following, may be obtained.

Proposition 7 If $G$ contains a vertex $v$ such that $\gamma_{s}(G-v) \geq \gamma_{s}(G)$, then $G$ is p-stable for some $p \leq \operatorname{deg}(v)-1 \leq \Delta-1$. 
Proof: Let $v$ be a vertex of $G$ such that $\gamma_{s}(G-v) \geq \gamma_{s}(G)$ and let $H$ be the graph obtained by removing all edges from $G$ that are incident to $v$. Then $\gamma_{s}(H)=\gamma_{s}(G-v)+1 \geq \gamma_{s}(G)+1>\gamma_{s}(G)$. Hence it is possible to increase the secure domination number of $G$ by removing $\operatorname{deg}(v)$ edges from $G$, and so $G$ is $p$-stable for some $p \leq \operatorname{deg}(v)-1 \leq \Delta-1$.

The following corollaries are immediate consequences of Proposition 7 .

Corollary 5 If $G$ contains a vertex $v$ such that $\gamma_{s}(G-v) \geq \gamma_{s}(G)$, then $G$ is $p$-stable for some $p \leq n-2$.

Corollary 6 If $G$ is an $(n-2)$-stable graph, then $\gamma_{s}(G-v)<\gamma_{s}(G)$ for any non-universal vertex $v$ of $G$.

Although a vertex $v$ for which $\gamma_{s}(G-v) \geq \gamma_{s}(G)$ may often be found in a graph $G$, this requirement is not necessary for the result of Corollary 5 . For example, the complete bipartite graph $\mathcal{K}_{4,4}$ has no such vertex, yet it is possible to prove the following result.

Proposition $8 \mathcal{K}_{4,4} \in \mathcal{S}_{8}^{6}$.

The proof of Proposition 8 is rather lengthy, consists of many cases and is not particularly instructive. The proof is therefore omitted here, but may be found in Appendix A. The result of Proposition 8 seems to be an isolated instance. The graph in Figure 4 for example, is a member of $\mathcal{K}_{r, r}-2(r-1) e$ which has no secure dominating set of cardinality 4 . This shows that Proposition 8 cannot be generalised to $\mathcal{K}_{r, r}$ and hence that $\mathcal{K}_{r, r} \notin \mathcal{S}_{n}^{n-2}$ for all $n=2 r$, where $r>4$.

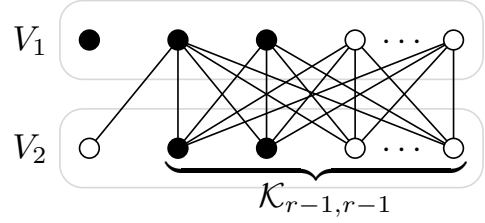

Fig. 4: A member of $\mathcal{K}_{r, r}-2(r-1) e$ which has no secure dominating set of cardinality 4 . A minimum secure dominating set of cardinality 5 is indicated by the solid vertices.

The following result is a similarly isolated case.

Proposition $9 \mathcal{K}_{3,3,3} \in \mathcal{S}_{9}^{7}$.

The proof of Proposition 9 is again rather lengthy and may be found in Appendix B. The graph in Figure 5 is a member of $\mathcal{K}_{r, r, r}-(3 r-2) e$ which has no secure dominating set of cardinality 3 and hence $\mathcal{K}_{r, r, r} \notin \mathcal{S}_{n}^{n-2}$ for $n=3 r$, where $r \geq 3$. This shows that the result of Proposition 9 cannot be generalised to $\mathcal{K}_{r, r, r}$.

\subsection{A lower bound on $\omega_{n}$}

We next turn our attention to establishing a lower bound on $\omega_{n}$.

Proposition 10 (Stability of stars) For any integer $n \geq 2, \mathcal{K}_{1, n-1} \in \mathcal{S}_{n}^{n-2}$. 


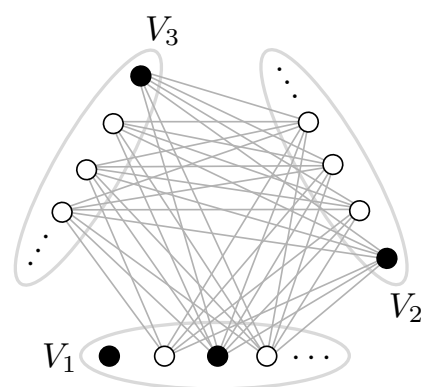

Fig. 5: A member of $\mathcal{K}_{r, r, r}-(3 r-2) e$ which has no secure dominating set of cardinality 3 . A minimum secure dominating set of cardinality 4 is indicated by the solid vertices.

Proof: The result is an immediate consequence of [4, Theorem 3], which states that

$$
C_{p}\left(\mathcal{K}_{1, n-1}\right)= \begin{cases}0 & \text { if } 0 \leq p \leq n-2 \\ 1 & \text { if } p=n-1\end{cases}
$$

for the star graph $\mathcal{K}_{1, n-1}$ of order $n$.

We therefore have the following corollary.

Corollary $7 \omega_{n} \geq n-2$ for all $n \geq 2$.

Our next result disqualifies large partitions of any sufficiently large independent set of a graph from forming part of a minimum secure dominating set of the graph.

Proposition 11 Let $G$ be a graph for which $\gamma_{s}(G)=k$. If $G$ contains an independent set $S$ of cardinality at least $k+1$, then every minimum secure dominating set of $G$ contains at most $k-2$ vertices of $S$.

Proof: By contradiction. Suppose $G$ contains an independent set $S$ of cardinality at least $k+1$ and let $X$ be a minimum secure dominating set of $G$. There are two cases to consider:

Case $i:|X \cap S|=k-1$. In this case $X$ contains a single vertex, $x$ (say), of $V(G)-S$ and there are at least two vertices, $y$ and $z$ (say), in the set $S-X$. Then $x$ is adjacent to both $y$ and $z$ (for otherwise $y$ and $z$ are not dominated). However, since $y$ and $z$ are nonadjacent, they are not defended by $x$. But clearly $y$ and $z$ are also not defended by any vertex in $X-\{x\}$, a contradiction.

Case ii: $|X \cap S|=k$. In this case $X \subset S$. But then no vertex in $S-X$ is dominated, a contradiction. These contradictions show that $|X \cap S| \leq k-2$, as required.

It follows from the contrapositive of the special case where $k=2$ in Proposition 11 that the secure domination number of the graph

$$
H=\mathcal{K}_{1, \underbrace{3,3, \ldots, 3}_{\text {eterms }}}
$$

is at least 3. But since $H \in \mathcal{K}_{n}-3 \ell e=\mathcal{K}_{n}-(n-1) e=\left(\mathcal{K}_{n}-e\right)-(n-2) e$, for $n=3 \ell+1$, it follows that $\mathcal{K}_{n}-e$ is not $p$-stable for any $p \geq n-2$ if $n \equiv 1(\bmod 3)$. Therefore, $\mathcal{K}_{n}-e \notin \mathcal{S}_{n}^{n-2}$ if $n \equiv 1(\bmod 3)$. 


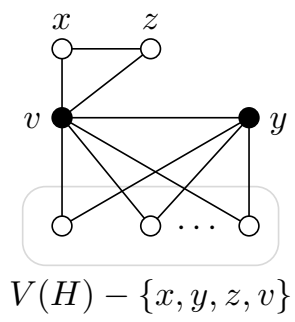

Fig. 6: A subgraph of $H$ for which $x, y, z$ are three consecutive vertices on a cycle of $\bar{H}$.

Despite this counter example, we next show that the graph class $\mathcal{S}_{n}^{n-2}$ nevertheless contains the graph $\mathcal{K}_{n}-e$ if $n \equiv 0,2(\bmod 3)$.

\section{Proposition 12}

If $n \geq 3$ is a natural number such that $n \equiv 0,2(\bmod 3)$, then $\mathcal{K}_{n}-e \in \mathcal{S}_{n}^{n-2}$.

Proof: For any integer $n \geq 3$, the graph $\mathcal{K}_{n}-e$ contains a vertex $v$ of degree $n-1$. Removal of the $n-1$ edges incident to $v$ yields the graph $\mathcal{K}_{1} \cup \mathcal{K}_{n-1}-e$. Since $\gamma_{s}\left(\mathcal{K}_{n}-e\right)=\gamma_{s}\left(\mathcal{K}_{n-1}-e\right)=2$, but $\gamma_{s}\left(\mathcal{K}_{1} \cup \mathcal{K}_{n-1}-e\right)=1+2=3$, it therefore follows that

$$
\mathcal{K}_{n}-e \text { is not } p \text {-stable for any integer } p \geq n-1 .
$$

Suppose now that $H \in \mathcal{K}_{n}-(n-1) e$. Then it follows by the pigeonhole principle that there is one vertex of $H$ with degree at least $n-2$ and another vertex of $H$ of degree at least $n-3$. We consider three cases:

Case i: At least one vertex of $H$ has degree $n-1$. There are three further subcases to consider.

Case i(a): At least two vertices of $H$ have degree $n-1$ each. In this case any two universal vertices form a secure dominating set of $H$.

Case i(b): Exactly one vertex of $H$ has degree $n-1$ and at least one vertex of $H$ has degree $n-2$. In this case the universal vertex and any vertex of degree $n-2$ form a secure dominating set of $H$.

Case i(c): Exactly one vertex of $H, v$ (say), has degree $n-1$, and no vertex of $H$ has degree $n-2$. In this case each vertex in $H-v$ has degree $n-3$. Since every vertex of $\bar{H}$ other than $v$ therefore has degree 2 , each component of $\bar{H}-v$ is a cycle. But since $n \not \equiv 1(\bmod 3)$, at least one of these cycles, $C$ (say), is not a triangle. Let $x, y$ and $z$ be three consecutive vertices on $C$. Then $x$ and $z$ are adjacent in $H$ and $y$ is adjacent to neither $x$ nor $z$ in $H$. But since the degree of $y$ in $H$ is $n-3, y$ is therefore adjacent to all the vertices in $V(H)-\{x, y, z\}$. Hence the graph in Figure 6 is a subgraph of $H$ and so $\{v, y\}$ is a secure dominating set of $H$, with $v$ defending itself as well as $x$ and $z$, and $y$ defending itself and all the vertices in $V(H)-\{x, y, z, v\}$. 
Case ii: No vertex of $H$ has degree $n-1$. In this case $\bar{H}$ has size $n-1$ and $\delta(\bar{H}) \geq 1$. Therefore at least one component of $\bar{H}$ is a nontrivial tree $T$. Since all trees are bipartite, let $V_{1}$ and $V_{2}$ be the partite sets of $T$. Then the set $\{x, y\}$, where $x \in V_{1}$ and $y \in V_{2}$, is a secure dominating set of $H$, since $x$ defends all the vertices in $V_{1}, y$ defends all the vertices in $V_{2}$, and both $x$ and $y$ defend the vertices in $V(H)-V_{1}-V_{2}$.

In both the above cases $\gamma_{s}(H)=2$ and hence $\mathcal{K}_{n}-e$ is $p$-stable for some $p \geq n-2$. The result therefore follows from (1).

It follows from Propositions 8,12 that the class of graphs

$$
\Lambda_{n}= \begin{cases}\left\{\mathcal{K}_{1,7}, \mathcal{K}_{8}-e, \mathcal{K}_{4,4}\right\} & \text { if } n=8 \\ \left\{\mathcal{K}_{1,8}, \mathcal{K}_{9}-e, \mathcal{K}_{3,3,3}\right\} & \text { if } n=9 \\ \left\{\mathcal{K}_{1, n-1}\right\} & \text { if } n \equiv 1(\bmod 3) \\ \left\{\mathcal{K}_{1, n-1}, \mathcal{K}_{n}-e\right\} & \text { otherwise }\end{cases}
$$

is a subset of $\mathcal{S}_{n}^{n-2}$ for all $n \geq 3$. We are unaware of any graphs in the class $\mathcal{S}_{n}^{n-2} \backslash \Lambda_{n}$ and conjecture as follows.

Conjecture $2 \mathcal{S}_{n}^{n-2}=\Lambda_{n}$ for all $n \geq 3$.

Our final result of this section shows that the validity of Conjecture 2 would imply the validity of Conjecture 1

Proposition 13 If $\Lambda_{n}=\mathcal{S}_{n}^{n-2}$, then $\omega_{n}=n-2$.

Proof: By contradiction. Suppose $\Lambda_{n}=\mathcal{S}_{n}^{n-2}$, but assume, to the contrary, that $\mathcal{S}_{n}^{n-1} \neq \emptyset$. Let $G \in$ $\mathcal{S}_{n}^{n-1}$. Then it follows from Proposition 3 that there is a member $H \in G-1 e$ of the class $\mathcal{S}_{n}^{n-2}$ such that $\gamma_{s}(G)=\gamma_{s}(H)$. There are three cases to consider:

Case i: $n \neq 8,9$. In this case $H \neq \mathcal{K}_{1, n-1}$, because $\gamma_{s}\left(\mathcal{K}_{1, n-1}\right)=n-1>n-2=\gamma_{s}\left(\mathcal{K}_{1, n-1}+e\right)$ as a result of the triangle in $\mathcal{K}_{1, n-1}+e$. Furthermore, $H \nsubseteq \mathcal{K}_{n}-e$, because $\gamma_{s}\left(\mathcal{K}_{n}-e\right)=2>$ $1=\gamma_{s}\left(\mathcal{K}_{n}\right)$. We therefore have a contradiction in this case.

Case ii: $n=8$. In this case, additionally $H \neq \mathcal{K}_{4,4}$, because $\gamma_{s}\left(\mathcal{K}_{4,4}\right)=4$ by [9. Proposition 11], yet $\Psi^{\prime}(2,1,4,0,0)$ is a certificate showing that $\gamma_{s}\left(\mathcal{K}_{4,4}+e\right) \leq 3$ [5. Proposition 3]. This is again a contradiction.

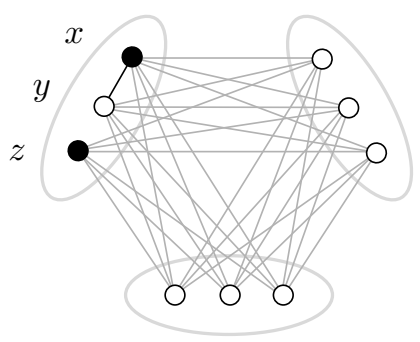

Fig. 7: The graph $\mathcal{K}_{3,3,3}+e$, which admits $\{x, y\}$ as secure dominating set. 
Case iii: $n=9$. In this case, additionally $H \neq \mathcal{K}_{3,3,3}$, because $\gamma_{s}\left(\mathcal{K}_{3,3,3}\right)=3$ by [9, Proposition 11], yet $\mathcal{K}_{3,3,3}+e$ is isomorphic to the graph in Figure 7. which admits $\{x, y\}$ as secure dominating set, showing that $\gamma_{s}\left(\mathcal{K}_{3,3,3}+e\right) \leq 2$, yet again a contradiction.

The above contradictions show that $\mathcal{S}_{n}^{n-1}=\emptyset$ and hence that $\omega_{n} \leq n-2$. The desired result therefore follows from Corollary 7

\section{Paths and Cycles}

In this section we consider the stability of paths and cycles. We start with paths.

\section{Proposition 14 (Stability of paths)}

The only paths that are 1 -stable are $\mathcal{P}_{3}$ and paths of the form $\mathcal{P}_{5+7 \ell}$ for some $\ell \in \mathbb{N}_{0}$. All other paths are 0-stable.

Proof: It is easily verified exhaustively that the paths $\mathcal{P}_{2}$ and $\mathcal{P}_{4}$ are 0 -stable, and that the paths $\mathcal{P}_{3}$ and $\mathcal{P}_{5}$ are 1 -stable. Suppose, therefore, that $n \geq 6$. It follows from [9. Theorem $12(\mathrm{~d})]$ that $\gamma_{s}\left(\mathcal{P}_{n}\right)=\lceil 3 n / 7\rceil$. Removal of the fifth edge from $\mathcal{P}_{n}$ yields the forest $\mathcal{P}_{5} \cup \mathcal{P}_{n-5}$ for which

$$
\gamma_{s}\left(\mathcal{P}_{5} \cup \mathcal{P}_{n-5}\right)=\left\lceil\frac{3(5)}{7}\right\rceil+\left\lceil\frac{3(n-5)}{7}\right\rceil=3+\left\lceil\frac{3 n}{7}-2-\frac{1}{7}\right\rceil=1+\left\lceil\frac{3 n-1}{7}\right\rceil,
$$

again by [9. Theorem 12(d)]. Therefore $\gamma_{s}\left(\mathcal{P}_{5} \cup \mathcal{P}_{n-5}\right)>\gamma_{s}\left(\mathcal{P}_{n}\right)$, unless $3 n \equiv 1(\bmod 7)$, which has the unique solution $n \equiv 5(\bmod 7)$. For any $n \neq 7 \ell+5$, the path $\mathcal{P}_{n}$ is therefore 0 -stable.

If $n=7 \ell+5$ for some $\ell \in \mathbb{N}$, then $\gamma_{s}\left(\mathcal{P}_{n}\right)=\lceil 3 n / 7\rceil=3 \ell+3$. Removal of the $k$-th edge from $\mathcal{P}_{n}$ yields the forest $\mathcal{P}_{k} \cup \mathcal{P}_{n-k}$ for which

$$
\gamma_{s}\left(\mathcal{P}_{k} \cup \mathcal{P}_{n-k}\right)=\left\lceil\frac{3 k}{7}\right\rceil+\left\lceil\frac{3(7 \ell+5-k)}{7}\right\rceil=\left\lceil\frac{3 k}{7}\right\rceil+3 \ell+2+\left\lceil\frac{1-3 k}{7}\right\rceil .
$$

Since $\gamma_{s}\left(\mathcal{P}_{n}\right) \leq \gamma_{s}\left(\mathcal{P}_{k} \cup \mathcal{P}_{n-k}\right)$, it follows that $\lceil 3 k / 7\rceil+\lceil(1-3 k) / 7\rceil \geq 1$. But by taking $a=3 k$ and $b=7$ in the identity $\lceil a / b\rceil+\lceil(1-a) / b\rceil \leq 1$, which holds for any $a, b \in \mathbb{Z}$ with $b \neq 0$, it follows that $\lceil 3 k / 7\rceil+\lceil(1-3 k) / 7\rceil \leq 1$, so that, in fact, $\lceil 3 k / 7\rceil+\lceil(1-3 k) / 7\rceil=1$. We conclude that $\gamma_{s}\left(\mathcal{P}_{n}\right)=\gamma_{s}\left(\mathcal{P}_{k} \cup \mathcal{P}_{n-k}\right)$ for any $k \in\{1, \ldots, n-1\}$ if $n=7 \ell+5$, showing that $\mathcal{P}_{n}$ is $p$-stable for some $p \geq 1$ in this case.

However, removal of the first and second edges from $\mathcal{P}_{7 \ell+5}$ yields the forest $2 \mathcal{P}_{1} \cup \mathcal{P}_{7 \ell+3}$ for which $\gamma_{s}\left(2 \mathcal{P}_{1} \cup \mathcal{P}_{7 \ell+3}\right)=2\lceil 3 / 7\rceil+\lceil 3(7 \ell+3) / 7\rceil=3 \ell+4>\gamma_{s}\left(\mathcal{P}_{n}\right)$, showing that $\mathcal{P}_{7 \ell+5}$ is not $p$-stable for any $p>1$. We therefore conclude that $\mathcal{P}_{7 \ell+5}$ is 1 -stable.

The stability results for cycles other than $\mathcal{C}_{3}$ follow immediately from Proposition 14 upon realisation that any edge removal from such a cycle produces a path of the same order. The cycle $\mathcal{C}_{3}$ is an exception merely because it is also a complete graph.

\section{Proposition 15 (Stability of cycles)}

The only cycles that are 2 -stable are cycles of the form $\mathcal{C}_{5+7 \ell}$ for some $\ell \in \mathbb{N}_{0}$. All other cycles, except $\mathcal{C}_{3}$, are 1-stable. Finally, $\mathcal{C}_{3}$ is 0 -stable. 


\section{Further work}

We propose the following three problems for future work:

1. In $\S 3$ we showed how the graph classes $\mathcal{S}_{n}^{1}, \ldots, \mathcal{S}_{n}^{\omega_{n}}$ can be computed inductively from the class $\mathcal{S}_{n}^{0}$ for all $n \geq 2$. We therefore propose the design of a construction process for the graph class $\mathcal{S}_{n}^{0}$ (Problem 1) as further work.

2. In $\S 4$ we showed that the graph class $\Lambda_{n}$ in 2 is a subclass of $\mathcal{S}_{n}^{n-2}$ for all $n \geq 2$. We believe that $\mathcal{S}_{n}^{n-2}=\Lambda_{n}$ for all $n \geq 2$ (Conjecture 2), but are unable to prove this. We therefore propose the proof of Conjecture 2 or, alternatively, demonstration of a graph in the class $\mathcal{S}_{n}^{n-2} \backslash \Lambda_{n}$ as a problem for future research.

3. Although the proof of Conjecture 2 would establish the truth of Conjecture 1 (by Proposition 13, it may of course be possible that Conjecture 1 is true even if Conjecture 2 is false. We therefore propose the resolution of Conjecture 1 as an independent problem.

\section{Acknowledgements}

The anonymous referees are thanked for their constructive criticism which has improved the quality of the paper. Research towards this paper was financially supported by the South African National Research Foundation (GUNs 70593, 77248 and 81558). The last author was additionally supported by a grant from Sub-committee A of Stellenbosch University's Research Committee.

\section{References}

[1] AP Burger, EJ Cockayne, WR Gründlingh, CM Mynhardt, JH VAn VuUren \& W Winterbach, 2004. Finite order domination in graphs, Journal of Combinatorial Mathematics and Combinatorial Computing, 49, 159-175.

[2] AP Burger, EJ Cockayne, WR Gründlingh, CM Mynhardt, JH VAn VuURen \& W Winterbach, 2004. Infinite order domination in graphs, Journal of Combinatorial Mathematics and Combinatorial Computing, 50, 179-194.

[3] AP Burger, AP DE Villiers \& JH VAN VuUREn. Edge criticality in secure graph domination, Discrete Applied Mathematics, submitted.

[4] AP Burger, AP DE Villiers \& JH VAn VuURen, 2014. The cost of edge failure with respect to secure graph domination, Utilitas Mathematica, 95, 329-339.

[5] AP Burger, AP DE VILliers \& JH VAN VUUREN. On minimum secure dominating sets of graphs, Quaestiones Mathematicae, submitted.

[6] AP Burger, MA Henning \& JH VAn VuURen, 2008. Vertex covers and secure domination in graphs, Quaestiones Mathematicae, 31(2), 163-171.

[7] EJ COCKAYNE, 2007. Irredundance, secure domination and maximum degree in trees, Discrete Mathematics, 307, 12-17.

[8] EJ Cockayne, O Favaron \& CM Mynhardt, 2003. Secure domination, weak roman domination and forbidden subgraphs, Bulletin of the Institute of Combinatorics and its Applications, 39, 87-100.

[9] EJ Cockayne, PJP Grobler, WR Gründlingh, J Munganga \& JH VAn Vuuren, 2005. Protection of a graph, Utilitas Mathematica, 67, 19-32. 
[10] PJP Grobler \& CM Mynhardt, 2009. Secure domination critical graphs, Discrete Mathematics, 309, 5820-5827.

[11] TW Haynes, ST Hedetniemi \& PJ Slater, 1998. Fundamentals of domination in graphs, Marcel Dekker, New York (NY).

[12] CM Mynhardt, HC SWART \& E Ungerer, 2005. Excellent trees and secure domination, Utilitas Mathematica, 67, 255-267.

[13] J SieK, LQ LeE \& A Lumsdaine, 2014. Boost graph library, [Online], [Cited: April 10 ${ }^{\text {th }}$, 2014], Available from http://www.boost.org/libs/graph/

\section{Appendix A: Proof of Proposition 8}

Suppose $V_{1}$ and $V_{2}$ are the partite sets of $\mathcal{K}_{4,4}$ and let $H$ be a subgraph of $\mathcal{K}_{4,4}$ that is isomorphic to a member of $\mathcal{K}_{4,4}-6 e$. Since $H \subseteq \mathcal{K}_{4,4}$ and $\gamma_{s}\left(\mathcal{K}_{4,4}\right)=4$ [9. Proposition 10],

$$
\gamma_{s}(H) \geq 4
$$

We show, by considering a number of cases, that, in fact, $\gamma_{s}(H)=4$.

The largest subgraph of $\mathcal{K}_{4,4}$ containing a vertex $v \in V_{1}$ with $d \in\{0,1,2,3,4\}$ private neighbours $u_{1}, \ldots, u_{d} \in$ $V_{2}$ is obtained by joining $v$ to all vertices in $V_{2}$ and joining each of the three vertices in $V_{1}-\{v\}$ to each of the $4-d$ vertices in $V_{2}-\left\{u_{1}, \ldots, u_{d}\right\}$. Because this subgraph has size $4+3(4-d)=16-3 d$ and $H$ has size 10 , it follows that $d \leq 2$ (i.e. $H$ has no vertex with more than two private neighbours). Moreover, if $H$ has a vertex with two private neighbours, then it has exactly one such vertex, $v$ (say), and $H$ is necessarily isomorphic to the graph in Figure 8 (a). In this case $H$ has the secure dominating set of cardinality 4 indicated by the solid vertices in the figure, showing that $\gamma_{s}(H)=4$ by 3 .

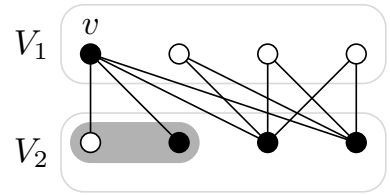

(a)

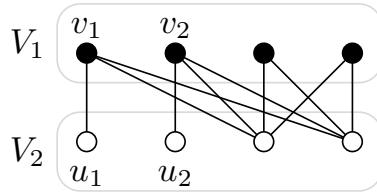

(b)

Fig. 8: The graph $H$ (a) with a vertex with two private neighbours, and (b) two vertices, each with exactly one private neighbour.

Suppose, therefore, that no vertex in $H$ has more than one private neighbour and let the number of vertices of $H$ with exactly one private neighbour be $k \in\{0,1,2,3,4\}$. The largest subgraph of $\mathcal{K}_{4,4}$ containing $k$ vertices $v_{1}, \ldots, v_{k} \in V_{1}$, each with one private neighbour, is obtained by joining $v_{i}$ to a private neighbour $u_{i} \in V_{2}$ for each $i=1, \ldots, k$ and by joining each vertex in $V_{1}-\left\{v_{1}, \ldots, v_{k}\right\}$ to all vertices in $V_{2}-\left\{u_{1}, \ldots, u_{k}\right\}$. Because this subgraph of $\mathcal{K}_{4,4}$ has size $k+4(4-k)=16-3 k$ and $H$ has size 10 , it follows that $k \leq 2$. Furthermore, if $H$ has exactly two vertices, each with exactly one private neighbour, then $H$ is necessarily isomorphic to the graph in Figure 8 b). In this case $H$ again has a secure dominating set of cardinality 4 , as indicated by the solid vertices in the figure, showing that $\gamma_{s}(H)=4$ by 3 .

We may therefore assume that exactly one vertex of $H, w$ (say), has at most one private neighbour and that the remaining vertices of $H$ have no private neighbours. Furthermore, since it requires at least $4+3=7$ edge removals from $\mathcal{K}_{4,4}$ to isolate two vertices of the graph, $H$ has at most one isolated vertex. Hence we have four final cases to consider: 
Case $i$ : $H$ has one isolated vertex, $x$ (say), and $w$ has one private neighbour $w^{\prime}$. In this case $x$ and $w^{\prime}$ cannot be in the same partite set, because the largest subgraph satisfying these conditions has size 9, as shown in Figure 9 (a). There is, however, only one subgraph of size 10 of $\mathcal{K}_{4,4}$ in which $x$ and $w^{\prime}$ are in different partite sets, as shown in Figure 9 b). In this case $H$ yet again has a secure dominating set of cardinality 4 , as indicated by the solid vertices in the figure, showing that $\gamma_{s}(H)=4$ in view of 3 .

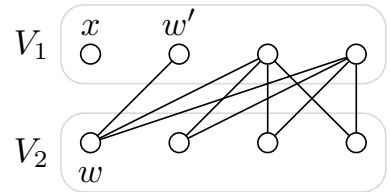

(a)

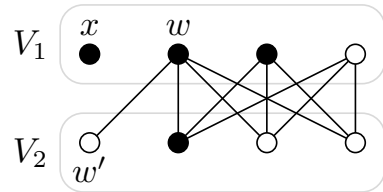

(b)

Fig. 9: The graph $H$ with one isolated vertex $x$, and one vertex $w$ which has one private neighbour $w^{\prime}$, where (a) $x$ and $w^{\prime}$ are in the same partite set, and (b) $x$ and $w^{\prime}$ are in different partite sets.

Case ii: $H$ has one isolated vertex, ( $x$ say), but no vertex with a private neighbour. Suppose $x \in V_{1}$ and let $v_{1}, v_{2}$ and $v_{3}$ be the other vertices in $V_{1}$. Since $\operatorname{deg}\left(v_{1}\right)+\operatorname{deg}\left(v_{2}\right)+\operatorname{deg}\left(v_{3}\right)=10$, it follows by the pigeonhole principle that at least one vertex in $V_{1}$ has degree 4 ; suppose this vertex is $v_{1}$. Then, since $\operatorname{deg}\left(v_{2}\right)+\operatorname{deg}\left(v_{3}\right)=6$, it follows by the pigeonhole principle that at least one of $v_{2}$ or $v_{3}$ has degree at least 3 ; suppose this vertex is $v_{2}$ and let the common neighbours of $v_{1}$ and $v_{2}$ be $w_{1}, w_{2}$ and $w_{3}$. Then the remaining vertex $w_{4}$ in $V_{2}$ is adjacent to either $v_{2}$ or $v_{3}$, for otherwise it would be a private neighbour of $v_{1}$. If $w_{4}$ is adjacent to $v_{2}$, then $H$ contains the subgraph in Figure 10 a), in which case $v_{3}$ is adjacent to exactly two vertices in $V_{2}, w_{i}$ and $w_{j}$ (say). But then $H$ has the secure dominating set $\left\{x, v_{1}, w_{i}, w_{j}\right\}$ of cardinality 4 . If, however, $w_{4}$ is adjacent to $v_{3}$, then $H$ contains the subgraph in Figure 10 b) which also has a secure dominating set of cardinality 4 , as indicated by the solid vertices in the figure. In both cases, therefore, $\gamma_{s}(H)=4$ by 3 .

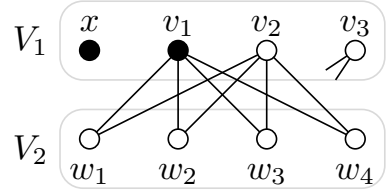

(a)

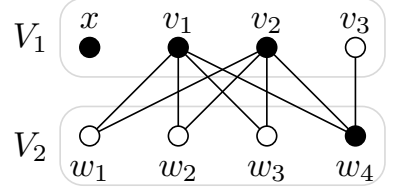

(b)

Fig. 10: Subgraphs of $H$ with an isolated vertex, ( $x$ say), but where no vertex has a private neighbour and where (a) $v_{3}$ is adjacent to $w_{i}$ and $w_{j}$, and (b) $v_{3}$ is adjacent to $w_{4}$.

Case iii: $H$ has no isolated vertices, and $w$ has one private neighbour $w^{\prime}$. Suppose $w \in V_{1}$ and let $v_{1}, v_{2}$ and $v_{3}$ be the other vertices in $V_{1}$. Since $\operatorname{deg}(w)+\operatorname{deg}\left(v_{1}\right)+\operatorname{deg}\left(v_{2}\right)+\operatorname{deg}\left(v_{3}\right)=10$, it follows by the pigeonhole principle that at least two vertices in $V_{1}$ have degree at least 3 . If $w$ has degree at least 3 , let $v_{1}$ be another vertex in $V_{1}$ with degree at least 3 . Then $H$ contains the subgraph in Figure 11 (a), in which case some vertex $u \in V_{2}$, which is adjacent to $v_{1}$, must be adjacent to at least one other vertex in $V_{1}, v_{3}$ (say). But then $H$ has the secure dominating set $\left\{w, v_{1}, v_{2}, v_{3}\right\}$ of cardinality 4 . If, however, $w$ has degree 2 , then two other vertices in $V_{1}, v_{1}$ and $v_{2}$ (say), have degree at least 3 . Then $H$ contains the subgraph in Figure 11 b) which also has a secure dominating set of cardinality 4 , as indicated by the solid vertices in the figure. In both cases, therefore, $\gamma_{s}(H)=4$ by 3 .

Case iv: $H$ has no isolated vertices and no vertices with private neighbours. Let $v_{1}, v_{2}, v_{3}$ and $v_{4}$ be the vertices in $V_{1}$. Since $\delta(H) \geq 2$, it follows by the pigeonhole principle that at least two vertices in $V_{1}, v_{1}$ and $v_{2}$ (say), have 


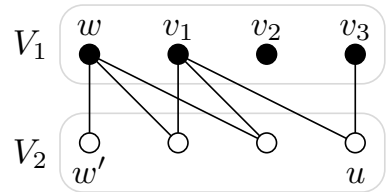

(a)

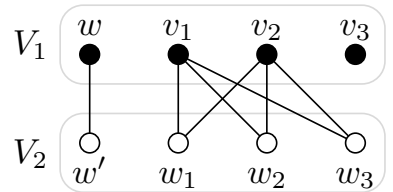

(b)

Fig. 11: The graph $H$ with no isolated vertices, where $w$ has one private neighbour $w^{\prime}$ and where (a) $w$ has degree at least 3 , and (b) $w$ does not have degree at least 3 .

degree 2. Furthermore, without loss of generality, either $v_{3}$ has degree 2 and $v_{4}$ has degree 4 , or else both $v_{3}$ and $v_{4}$ have degree 3 . If $v_{3}$ has degree 2 and $v_{4}$ has degree 4 , then $H$ contains the subgraph in Figure $12($ a), in which case $H$ has the secure dominating set $\left\{v_{1}, v_{2}, v_{3}, v_{4}\right\}$, because $N\left(v_{1}\right) \cup N\left(v_{2}\right) \cup N\left(v_{3}\right)=V_{2}$ in order to avoid private neighbours in $H$. In this case, therefore, $\gamma_{s}(H)=4$ by 3 .

Finally, if both $v_{3}$ and $v_{4}$ have degree 3 , then $N\left(v_{3}\right)=N\left(v_{4}\right)$ or $N\left(v_{3}\right) \neq N\left(v_{4}\right)$. If $N\left(v_{3}\right)=N\left(v_{4}\right)$, then there is a vertex $u \in V_{2}$ that is adjacent to neither $v_{3}$ nor $v_{4}$, in order to avoid private neighbours in $H$. Since $\delta(H) \geq 2, u$ is adjacent to $v_{1}$ and $v_{2}$, as shown in Figure $12 \mathrm{~b}$ ), in which case $H$ has the secure dominating set $\left\{v_{1}, v_{2}, v_{3}, v_{4}\right\}$ of cardinality 4 , showing that $\gamma_{s}(H)=4$ by 3 . If, however, $N\left(v_{3}\right) \neq N\left(v_{4}\right)$, then some vertex $u \in V_{2}$ is adjacent to $v_{3}$ but not to $v_{4}$. Since $\delta(H) \geq 2, u$ is adjacent to at least one other vertex in $V_{1}$. Assume, without loss of generality, that $u$ is adjacent to $v_{1}$. Then $H$ contains the subgraph in Figure 12 c), in which case $H$ has the secure dominating set $\left\{v_{1}, v_{2}, v_{3}, v_{4}\right\}$ of cardinality 4 , showing that $\gamma_{s}(H)=4$ by 3 .

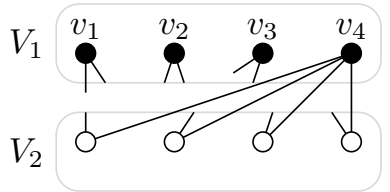

(a)

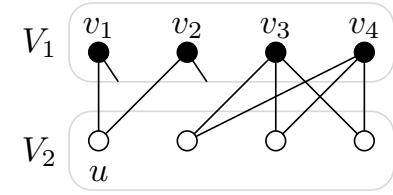

(b)

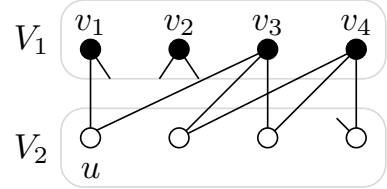

(c)

Fig. 12: The graph $H$ with no isolated vertices and no vertices with private neighbours, where (a) $\operatorname{deg}\left(v_{3}\right)=2$ and $\operatorname{deg}\left(v_{4}\right)=4$, (b) $\operatorname{deg}\left(v_{3}\right)=\operatorname{deg}\left(v_{4}\right)=3$ and $N\left(v_{3}\right)=N\left(v_{4}\right)$, and (c) $\operatorname{deg}\left(v_{3}\right)=\operatorname{deg}\left(v_{4}\right)=3$ and $N\left(v_{3}\right) \neq N\left(v_{4}\right)$.

From the above cases we conclude that $\gamma_{s}(H)=4$ and hence that $\mathcal{K}_{4,4}$ is $p$-stable for some $p \geq 6$. The subgraph $2 \mathcal{K}_{1} \cup \mathcal{K}_{3,3}$ of size $16-7=9$ of $\mathcal{K}_{4,4}$, however, has secure domination number $2+3=5$ [9. Proposition 10], showing that $\mathcal{K}_{4,4}$ is not $p$-stable for any $p \geq 7$.

\section{Appendix B: Proof of Proposition 9}

Suppose $V_{1}, V_{2}$ and $V_{3}$ are the partite sets of $\mathcal{K}_{3,3,3}$ and let $H$ be a subgraph of $\mathcal{K}_{3,3,3}$ that is isomorphic to a member of $\mathcal{K}_{3,3,3}-7 e$. Since $H \subseteq \mathcal{K}_{3,3,3}$ and $\gamma_{s}\left(\mathcal{K}_{3,3,3}\right)=3$ by Proposition [9 Proposition 11],

$$
\gamma_{s}(H) \geq 3 \text {. }
$$

It is shown, by considering a number of cases, that, in fact, $\gamma_{s}(H)=3$.

Let $V_{1}=\left\{u_{1}, u_{2}, u_{3}\right\}, V_{2}=\left\{v_{1}, v_{2}, v_{3}\right\}$ and $V_{3}=\left\{w_{1}, w_{2}, w_{3}\right\}$. It follows from the pigeonhole principle that at least four vertices in $H$ have degree at least 5 . Furthermore, two vertices of the same partite set of $H, u_{1}, u_{2} \in V_{1}$ (say), have degree at least 5 . Two cases are considered. 


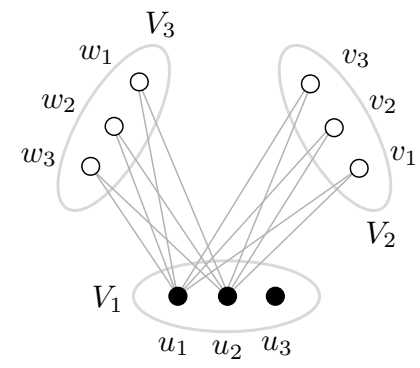

(a)

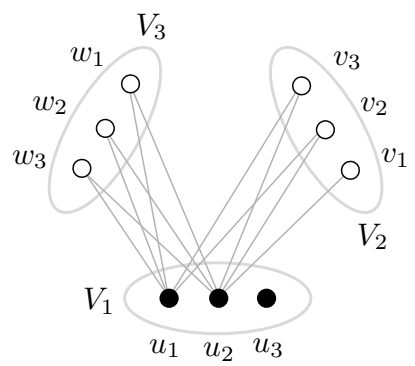

(b)

Fig. 13: Subgraphs of $H$ with $\Delta(H)=6$, where (a) $\operatorname{deg}\left(u_{1}\right)=\operatorname{deg}\left(u_{2}\right)=6$, with both $u_{1}$ and $u_{2}$ defending $V_{1} \cup V_{2}$, and (b) $\operatorname{deg}\left(u_{2}\right)=6$ and $\operatorname{deg}\left(u_{1}\right)=5$, with $u_{2}$ defending $v_{1}$, and $u_{1}$ defending $\left(V_{2}-\left\{v_{1}\right\}\right) \cup V_{3}$. A minimum secure dominating set is indicated by the solid vertices in each case.

Case $i: \Delta(H)=6$. If both $u_{1}$ and $u_{2}$ have degree 6 , then $H$ contains the subgraph in Figure 13 a), in which case the partite set $V_{1}$ is a secure dominating set of $H$ (of cardinality 3 ). Suppose then that only one vertex, $u_{2}$ (say), has degree 6 , while $u_{1}$ has degree 5 . Then $H$ contains the subgraph in Figure 13 (b), in which case the partite set $V_{1}$ is yet again a secure dominating set of $H$ (of cardinality 3). In both cases, therefore, $\gamma_{s}(H)=3$ by (4).

Case ii: $\Delta(H) \leq 5$. In this case at least two vertices, $u_{1}$ and $u_{2}$ (say), of $V_{1}$ have degree 5 . It follows by the pigeonhole principle that $\delta(H) \geq 1$. However, if $\delta(H)=1$, then $H$ is isomorphic to either the graph in Figure 14(a), the graph in Figure 14 (b) or the graph in Figure 14 c). In all of these cases $\gamma_{s}(H)=3$. Assume, therefore, that $\delta(H) \geq 2$. There are two subcases to consider:

Case ii(a): $N\left(u_{1}\right)=N\left(u_{2}\right)$. In this case exactly one vertex, $v_{1}$ (say), in $V_{2} \cup V_{3}$ is adjacent to neither $u_{1}$ nor $u_{2}$. If $v_{1}$ and $u_{3}$ are adjacent, then $H$ contains the subgraph in Figure $15(\mathrm{a})$, in which case $V_{1}$ is a secure dominating set of $H$. Suppose, therefore, that $v_{1}$ and $u_{3}$ are not adjacent. Since $v_{1}$ is not adjacent to any vertex in $V_{1} \cup V_{2}$ and $u_{3}$ is not adjacent to any vertex in $V_{1} \cup\left\{v_{1}\right\}$, and since $\operatorname{deg}\left(v_{1}\right)+\operatorname{deg}\left(u_{3}\right) \geq 7$, it follows that $u_{3}$ and $v_{1}$ share at least two common neighbours, $w_{1}$ and $w_{2}$ (say), in $V_{3}$, as shown in Figure 15 (b). Furthermore, since $\operatorname{deg}\left(v_{1}\right)+\operatorname{deg}\left(u_{3}\right) \leq 8$, there are at least eight edges between the vertices in $V_{2}$ and $V_{3}$. Hence, $v_{2}$ and $v_{3}$ are both adjacent to at least one of

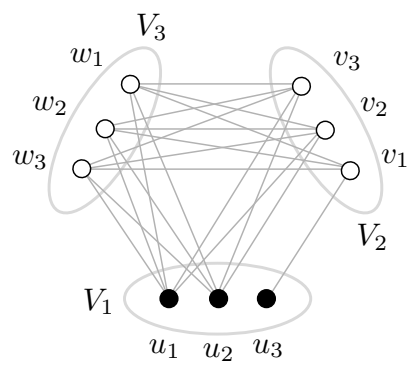

(a)

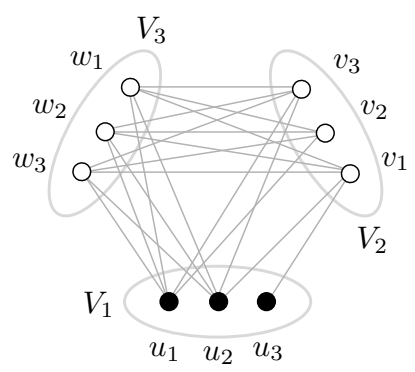

(b)

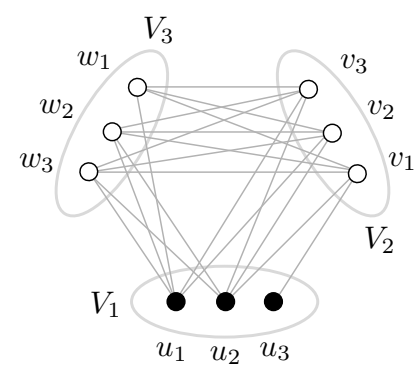

(c)

Fig. 14: The graph $H$ with $\Delta(H)=5$ and $\delta(H)=1$, with (a) $\operatorname{deg}\left(v_{1}\right)=4$, where $u_{3}$ defends $v_{1}$ and $u_{1}$ defends $V_{1} \cup\left(V_{2}-\left\{v_{1}\right\}\right)$, (b) $\operatorname{deg}\left(v_{1}\right)=5$ and $\operatorname{deg}\left(v_{2}\right)=4$, where $u_{1}$ defends $v_{3}$ and $u_{1}$ defends $V_{1} \cup\left(V_{2}-\left\{v_{1}\right\}\right)$, and (c) $\operatorname{deg}\left(v_{1}\right)=5$ and $\operatorname{deg}\left(w_{1}\right)=4$, where $u_{3}$ defends $v_{1}$ and $u_{1}$ defends $V_{1} \cup\left(V_{2}-\left\{v_{1}\right\}\right)$. A minimum secure dominating set is indicated by the solid vertices in each case. 


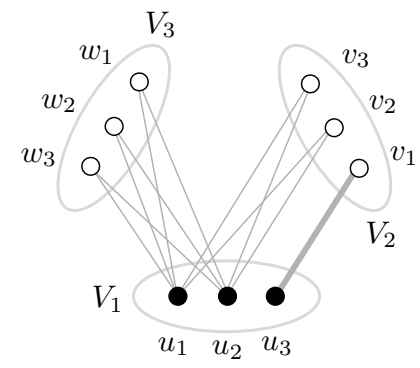

(a)

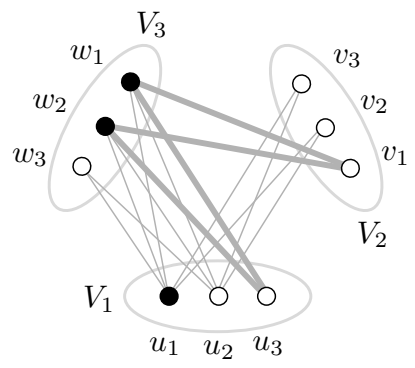

(b)

Fig. 15: Subgraphs of $H$ with $\Delta(H)=5$ and $\delta(H)=2$, where $u_{1}$ and $u_{2}$ have identical neighbourhoods and where (a) $u_{3}$ and $v_{1}$ are neighbours, with $u_{3}$ defending $v_{1}$, and both $u_{1}$ and $u_{2}$ defending the vertices in $\left(V_{2}-\left\{v_{1}\right\}\right) \cup V_{3}$, and (b) $u_{3}$ and $v_{1}$ are not neighbours, with $u_{1}$ defending $w_{3}$, and $w_{1}$ and $w_{2}$ both defending $\left\{u_{2}, u_{3}, v_{1}, v_{2}, v_{3}\right\}$. A minimum secure dominating set is indicated by the solid vertices in each case.

$w_{1}$ or $w_{2}$. In this case, $\left\{u_{1}, w_{1}, w_{2}\right\}$ is a secure dominating set of $H$. In this subcase, therefore, $\gamma_{s}(H)=3$ by (4).

Case $\mathrm{ii}(b): N\left(u_{1}\right) \neq N\left(u_{2}\right)$. In this case $u_{1}$ is adjacent to a vertex, $x$ (say), in $V_{2} \cup V_{3}$ to which $u_{2}$ is not adjacent and, similarly, $u_{2}$ is adjacent to a vertex, $y$ (say), in $V_{2} \cup V_{3}$ to which $u_{1}$ is not adjacent.

Suppose first that $x$ and $y$ are in the same partite set. It may be assumed, without loss of generality, that $x=v_{1}$ and $y=v_{2}$. Since $\delta(H) \geq 2, u_{3}$ is adjacent to at least two vertices in $V_{2} \cup V_{3}$. If $u_{3}$ is adjacent to either $v_{1}$ or $v_{2}$, then $V_{1}$ is a secure dominating set of $H$, as shown in Figure 16 a). If $u_{3}$ is adjacent to neither $v_{1}$ nor $v_{2}$, but $u_{3}$ shares a neighbour, $w_{1}$ (say), with either $v_{1}$ or $v_{2}$, then $\left\{u_{1}, u_{2}, w_{1}\right\}$ is a secure dominating set of $H$, as shown in Figure 16 b). Finally, if $u_{3}$ is adjacent to neither $v_{1}$ nor $v_{2}$, and $u_{3}$ shares no common neighbours with $v_{1}$ and $v_{2}$, then $u_{3}, v_{1}$ and $v_{2}$ are all adjacent to distinct vertices in $V_{3}$ and $u_{3}$ is also adjacent to $w_{3}$. It may be assumed, without loss of generality, that $v_{1}$ is adjacent to $w_{2}$ and that $v_{2}$ is adjacent to $w_{1}$. Then $V_{1}$ is a secure dominating set of $H$, as

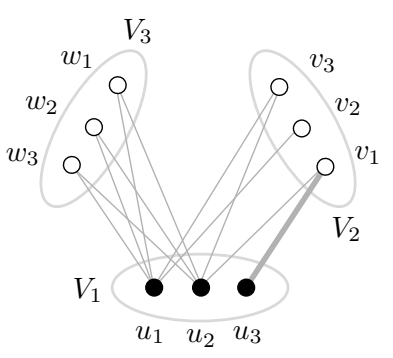

(a)

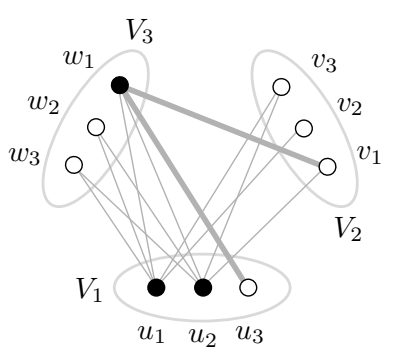

(b)

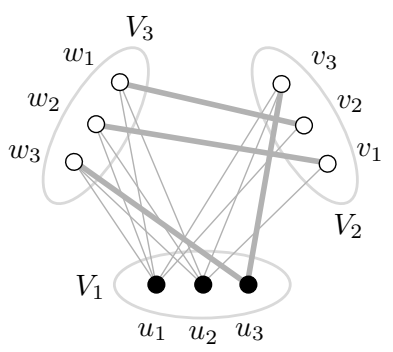

(c)

Fig. 16: Subgraphs of $H$ with $\Delta(H)=5$ and $\delta(H)=2$, where $u_{1}$ and $u_{2}$ do not have identical neighbourhoods and where (a) $u_{3}$ is adjacent to $v_{1}$, with $u_{3}$ defending $v_{1}$ and $u_{2}$ defending $\left(V_{2}-\left\{v_{1}\right\}\right) \cup V_{3}$, (b) $u_{3}$ is not adjacent to $v_{1}$ or $v_{2}$, but shares $w_{1}$ as common neighbour with them, in which case $w_{1}$ defends $u_{3}$, while $u_{2}$ defends $\left(V_{2}-\right.$ $\left.\left\{v_{2}, w_{1}\right\}\right) \cup V_{3}$ and $u_{2}$ defends $v_{2}$, and (c) $u_{3}$ is adjacent to neither $v_{1}$ nor $v_{2}$ and shares no common neighbour with $v_{1}$ or $v_{2}$, in which case $u_{3}$ defends $\left\{v_{3}, w_{3}\right\}, u_{2}$ defends $\left\{v_{1}, w_{2}\right\}$ and $u_{1}$ defends $\left\{v_{2}, w_{1}\right\}$. A minimum secure dominating set is indicated by the solid vertices in each case. 


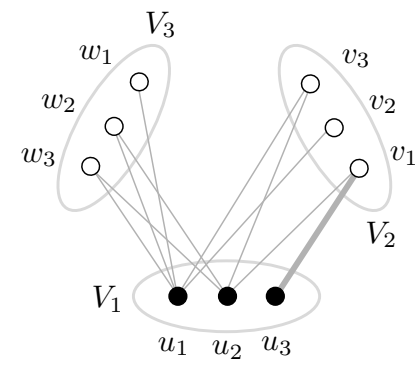

(a)

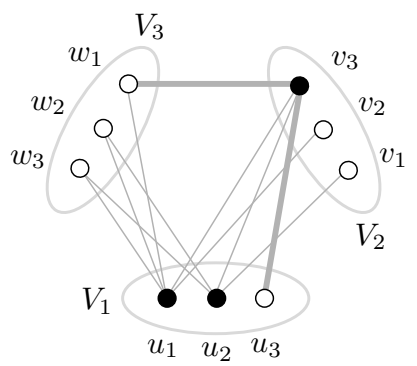

(b)

Fig. 17: Subgraphs of $H$ with $\Delta(H)=5$ and $\delta(H)=2$, where $u_{1}$ and $u_{2}$ do not have identical neighbourhoods and where (a) $u_{3}$ is adjacent to $v_{1}$, in which case $u_{3}$ defends $v_{1}$ and $u_{2}$ defends $\left(V_{2}-\left\{v_{1}, v_{2}\right\}\right) \cup V_{3}$, while $u_{1}$ defends $v_{2}$, and (b) $u_{3}$ is adjacent to neither $v_{1}$ nor $w_{1}$, but $u_{3}$ shares a neighbour $v_{3}$ with $w_{1}$, in which case $v_{3}$ defends $u_{3}, u_{2}$ defends $v_{1}$ and $u_{1}$ defends $\left(V_{2}-\left\{v_{1}, v_{3}\right\}\right) \cup V_{3}$. A minimum secure dominating set is indicated by the solid vertices in each case.

shown in Figure 16. (c). In all of these cases, therefore, $\gamma_{s}(H)=3$ by 4 .

Suppose $x \in V_{2}$ and $y \in V_{3}$. It may be assumed, without loss of generality, that $x=v_{1}$ and $y=w_{1}$. If $u_{3}$ is adjacent to either $v_{1}$ or $w_{1}$, or to both $v_{1}$ and $w_{1}$, then $V_{1}$ is a secure dominating set of $H$, as shown in Figure 17 (a). Suppose, therefore, that $u_{3}$ is adjacent to neither $v_{1}$ nor $w_{1}$, but that $u_{3}$ shares a neighbour, $z$ (say), with either $v_{1}$ or $w_{1}$. It may be assumed, without loss of generality, that $z=v_{3}$, in which case $\left\{u_{1}, u_{2}, v_{3}\right\}$ is a secure dominating set of $H$, as shown in Figure 17b). If $u_{3}$ is adjacent to neither $v_{1}$ nor $w_{1}$, and $u_{3}$ shares no common neighbours with $v_{1}$ and $w_{1}$, but $v_{1}$ and $w_{1}$ are adjacent, then $\operatorname{deg}\left(v_{1}\right)+\operatorname{deg}\left(w_{1}\right) \leq 6$, in which case $\Delta(H)=6$, a contradiction. Finally, suppose that $u_{3}$ is adjacent to neither $v_{1}$ nor $w_{1}, u_{3}$ shares no common neighbours with $v_{1}$ and $w_{1}$, and that $v_{1}$ and $w_{1}$ are not adjacent. It may be assumed, without loss of generality, that $v_{1}$ is adjacent to $w_{2}$ and that $w_{1}$ is adjacent to $w_{2}$. Then $\operatorname{deg}\left(v_{1}\right)+\operatorname{deg}\left(w_{1}\right)=4$, in which case $\Delta(H)=6$, again a contradiction. In both of the cases that did not lead to contradictions, however, $\gamma_{s}(H)=3$ by $(4)$.

From all of the above cases it is concluded that $\gamma_{s}(H)=3$ and hence that $\mathcal{K}_{3,3,3}$ is $p$-stable for some $p \geq 7$. Finally, the subgraph of $\mathcal{K}_{3,3,3}$ in Figure 18 has size $27-8=19$ and secure domination number 4 , showing that $\mathcal{K}_{3,3,3}$ is not stable for any $p \geq 8$.

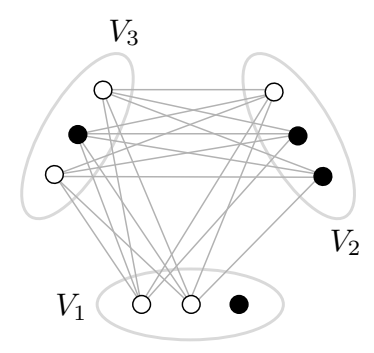

Fig. 18: A graph $H \subseteq \mathcal{K}_{3,3,3}-8 e$ for which $\gamma_{s}(H)=4$. A minimum secure dominating set is indicated by the solid vertices in each case. 